\title{
GADD153 as a novel molecular fingerprint for Sjögren's syndrome
}

\author{
Mahmood S. Mozaffari, Jun Yao Liu, Rafik Abdelsayed, Babak Baban \\ From EPMA-World Congress 2013 \\ Brussels, Belgium. 20-21 September 2013
}

\section{Scientific objective}

Sjögren's syndrome (SS) is a systemic autoimmune disease characterized by chronic focal leukocyte infiltration and inflammation of exocrine glands including salivary glands thereby resulting in persistent dry mouth and consequent increased risk for dental caries and periodontal disease. Assessment of leukocyte infiltration into minor salivary glands of lower lip biopsy samples is commonly used to aid with diagnosis of SS; however, this approach may identify $60-80 \%$ of those afflicted with the disease thereby requiring use of additional diagnostic tests/markers which relies on a better understanding of the pathogenesis of SS. Endoplasmic reticulum (ER) stress response is a pivotal regulator of inflammation and cell death. An integral component of ER stress-induced apoptosis is expression of the growth arrest- and DNA damage-inducible protein 153 (GADD153). Thus, we tested the hypothesis that SS is associated with increased salivary gland expression of GADD153 and increased leukocyte infiltration thereby contributing to inflammation and cell death.

\section{Technical approach/methods}

We utilized the non-obese diabetic (NOD) mice, a model of SS-like disease, in association with immunostaining and flow cytometry-based studies.

\section{Results/interpretation}

Salivary glands of NOD mice displayed a) increased GADD153 expression, b) foci of inflammatory cell infiltrates including $\mathrm{CD}^{+} \mathrm{T}$ and $\mathrm{CD} 19^{+} \mathrm{B}$ lymphocytes as well as M1 and M2 macrophages and c) increased proinflammatory interleukin (IL)-17 but reduced antiinflammatory cytokine, IL-10. These changes were accompanied with disruption of mitochondrial membrane potential and significant increase in apoptosis and necrosis of salivary gland cells of NOD than control mice. To establish the relevance of GADD153 for the human condition, archived lower lip biopsy samples of non-SS subjects and those with a diagnosis of SS were subjected to immunostaining. The results show intense GADD153 immunostaining in biopsy samples of SS compared to non-SS subjects. Collectively, the results indicate that GADD153 regulates inflammation and cell death in salivary gland in SS.

\section{Outlook/expert recommendations}

The present study has established the relevance and significance of GADD153 as a novel predictive and prognostic molecular fingerprint for SS. Further, assessment of minor salivary gland GADD153 expression could potentially be a more sensitive approach to help with diagnosis of SS, at early stage of the disease, than leukocyte infiltration. Importantly, subsequent studies should establish whether inhibition of GADD153 expression ameliorates signs and symptoms of SS for which effective treatment options remain elusive.

Published: 11 February 2014

doi:10.1186/1878-5085-5-S1-A110

Cite this article as: Mozaffari et al: GADD153 as a novel molecular

fingerprint for Sjögren's syndrome. EPMA Journal 2014 5(Suppl 1):A110.

\footnotetext{
* Correspondence: MMOZAFFA@gru.edu

Department of Oral Biology, College of Dental Medicine, Georgia Regents University, Augusta, Georgia 30912, USA
} 